\title{
Comparative study of the use of arthrocentesis and arthrocentesis in addition with platelet rich plasma in management of temporomandibular joint internal derangement
}

\author{
Original Yasser Nabil ${ }^{1}$, Ph.D., Heba Seleem², Ph.D., Nehal Hassan ${ }^{1}$, B.DS \\ Article \\ ${ }^{1}$ Assistant Professor and Head of Department of Oral and Maxillofacial Surgery, Specialized
Dental Teaching Hospital, Misr University of Science and Technology, ${ }^{2}$ Assistant Professor,
Department of Oral and Maxillofacial Surgery, Faculty of Dentistry, Ain-Shams University,
${ }^{3}$ Resident in Department of Oral and Maxillofacial Surgery, Specialized Dental Teaching
Hospital.
}

\begin{abstract}
Background: Disc derangement disorders of the TMJ are a group of intra-articular biomechanical disorders in which there is an abnormal relationship in the functional 'articular cartilaginous' condyle-disc complex.

Platelet-rich plasma (PRP) is a blood product that allows in a simple, low cost, and minimally invasive way to obtain a concentration of many growth factors when activated.

Aim: To compare the use of arthrocentesis alone and arthrocentesis in addition with platelet rich plasma in management of TMJ internal derangement.

Materials and Methods: fourteen patients with DDwOR were included in the study diagnosed according to The Research Diagnostic Criteria for Temporomandibular disorders (RDC/TMD) and divided into two groups as follow; seven patients underwent intra-articular injections of $2 \mathrm{ml}$ PRP after arthrocentesis (study group) and the other seven patients were treated by the conventional arthrocentesis using Ringer's solution (control group). The patients were clinically evaluated preoperatively and postoperatively at the intervals of 1 month and 6 months.

Results: The differences between the two groups in all the measured parameters were statistically insignificant throughout the postoperative period.

Conclusion: injection is a safe and effective method in the treatment of TMDs.
\end{abstract}

Key Words: Arthrocentesis, DDwOR, PRP, TMDs, TMJ

Received: 22 March 2019, Accepted: 25 April 2019

Corresponding Author: Yasser Nabil, Department of Oral and Maxillofacial Surgery, Specialized Dental Teaching Hospital, Misr University of Science and Technology, Cairo, Egypt, Tel.: 01001451772, E-mail: dryasser_nabil@yahoo.co.uk

ISSN: 2090-097X, January 2019, Vol. 10, No. 1

\section{INTRODUCTION}

TMJ is a complex synovial joint consisting of temporal bone, mandibular bone, articular disc, synovial membrane, and associated ligaments and muscles. It consists of two compartments the upper and lower compartments 1,2, this unique structure facilitates a mouth opening of $40-60 \mathrm{~mm}$ as measured between the upper and lower incisors where rotation of the condyle occurs in the lower compartment enables about $15-25 \mathrm{~mm}$ opening while the translation of the condyle with the disc along affords a mouth opening of 40-60 $\mathrm{mm}$ as well as lateral movements of up to $10 \mathrm{~mm}$, protrusive movement of up to $9 \mathrm{~mm}$ and retrusive movements of $1 \mathrm{~mm} .3$

TMDs are collection of medical and dental conditions affecting the joint and muscles of mastication, as well as contiguous tissue components ${ }^{[4]}$.

The RDC/TMD applies a dual-axis system to diagnose and classify patients with TMD. The physical axis 1 classify TMD patients into (I) myofascial pain, (II) disc displacement with/without reduction, and (III) arthralgia, osteoarthritis, and osteoarthrosis. The psychosocial axis 2 includes a 31-item questionnaire that assesses TMD-related pain and psychosocial factors ${ }^{[5]}$. This multiaxial approach allows better characterization of the patient from several standpoints ${ }^{[6]}$.

One of the most common types of disorders is internal derangement of the disc. It may present with a numerous of overlapping signs and symptoms including pain in the joint, limitation of mandibular movements (e.g., locking), TMJ sounds and occasionally headaches ${ }^{[7]}$.

The primary goal in the treatment of TMJ disorders is to alleviate pain and to restore mandibular function (mastication and speech) initially using conservative measures, which will resolve symptoms in over $80 \%$ patients $^{[8]}$. 
Surgical intervention is typically employed only after failure of non surgical treatment .

Arthrocentesis and TMJ arthroscopy have been found to be minimally invasive effective treatment for articular TMJ disorders by decreasing pain and increasing mandibular range of motion .

Arthrocentesis is the first-line surgical intervention in TMD/DDwoR patients who do not respond to conservative management ${ }^{[9,10]}$. It is a minimally invasive procedure which is considered as an intermediate treatment modality between non-invasive conservative and more invasive surgical interventions ${ }^{[11,12]}$.

Although the use of arthrocentesis in management of TMJ internal derangement proved reasonable degree of success ranging from 75 to $100 \%$. Intra-articular injection using different medication was used in combination with arthrocentesis to enhance the clinical outcome and improve long term results .

PRP is a natural concentrate of growth factors from blood. It is observed that these preparations had bone forming properties as well as anti-inflammatory and antibacterial properties ${ }^{[13]}$.

PRP therapy is based on the effects of GFs that promote changes in cell proliferation and regulate cellular metabolism. GFs have a vital role in modulating chondrogenic expression $^{[14]}$. PRP promotes healing through regeneration of degenerative changes in cartilage, bone, and synovial tissue ${ }^{[15]}$. In oral surgery, PRP is used to support bone regeneration in sinus lifts, cleft surgery, and jaw reconstruction, promotes healing of oroantral communications, bone defects, and cysts, and prevents further development of dry socket ${ }^{[16]}$.

While, articular cartilage has limited regenerative capacity due to its avascularity and low mitotic activity, some GFs especially TGF beta,basic fibroblast growth factor, and bone morphogenic protein show a positive effect on cartilage tissue regeneration ${ }^{[17,18]}$.

TGF- $\beta$ is one of the most important factor involved in the process of cartilage regeneration; its function includes increased chondrocyte phenotype expression ${ }^{[}$ ${ }^{19]}$ the chondrocyte differentiation of mesenchymal stem cells, matrix deposition and counteract with most of the suppressive effects of inflammatory mediators IL 1 on cartilage specific macromolecules synthesis ${ }^{[20]}$. PDGF also plays an important role in the maintenance of hyaline like chondrogenic phenotype, increases chondrocyte proliferation, upregulation of proteoglycan synthesis, and is a potent chemotactic factor for all cells of mesenchymal origin $^{[21]}$. IGF is another important cartilage anabolic factor and it has an important role in augmenting the effects of other growth factors found in cartilage ${ }^{[22]}$. Many other growth factors are involved in cartilage regeneration and metabolism, like FDG and HGF, and they may have chondroinductive actions, independently or more so with additive effects and synergistic interaction ${ }^{[23]}$.

This study was designed to investigate the effectiveness of arthrocentesis either alone or with subsequent intrajoint medications as PRP.

\section{MATERIALS AND METHODS}

Patients affiliated to our clinic were examined by using RDC/TMD as the basic research diagnostic system. Clinical head and neck examination was established to select participants of the study.

This clinical examination included Site of pain, pattern of jaw movement, measurement of the range of mouth opening, measurement of lateral excursions, measurement of protrusion and joint noises.

Cases were enrolled in the study as follows:

-Axis 1 group $2 b$ (disc displacement without reduction with limitation)

-Axis 1 group 2c (disc displacement without reduction without limitation)

Diagnosis was confirmed by a standardized preoperative MRI examination.

All the patients have undergone a period of conservative treatment which was 6 months. Patients who were not responsive to these non surgical treatments had been selected to our clinical trial. Informed written consents were obtained from them after explaining the nature of the procedure, duration of the postoperative follow up and the possible complications.

The study was conducted on 14 patients with a total of 20 joints). They were randomly assigned into two groups, each group contained 7 patients with a total of 10 joints ( 3 bilateral and 4 unilateral cases).

A control group receiving lavage and a study group receiving lavage with injection of PRP.

Each patient had completed a questionnaire evaluating TMJ pain, joint clicking, and maximum mouth opening. TMJ pain has been assessed using research diagnostic criteria (RDC), visual analog scale (VAS) in a $10 \mathrm{~cm}$ line with one end labeled with no pain and another end with severe pain. Maximum mouth opening (MMO), lateral excursion, protrusion was measured with millimeter scale and clicking sound was assessed by palpating the joint 
during opening and closing of mouth. The procedure was performed under general anasthesia

\section{Operative Procedure:}

A line was drawn from middle of the tragus to the outer canthus. Entrance points were located along the canthotragal line, where the first point was marked 10 $\mathrm{mm}$ from the middle of the tragus and $2 \mathrm{~mm}$ below the canthotragal line, it corresponds to the glenoid fossa. A second point corresponding to the articular eminence was marked $10 \mathrm{~mm}$ from the first point and $10 \mathrm{~mm}$ below the line.

A total of $150-200 \mathrm{ml}$ riger lactate solution was then injected under pressure through the first needle into the superior joint space while the second needle provided the outflow.

The outflow needle was then removed and $2 \mathrm{ml}$ platelet rich plasma was injected in the study group into the superior joint space through the inflow needle.

Patient's mandible was manipulated in vertical, protrusive and lateral excursions facilitating lysis of adhesions, distribution of the drug, and freeing disc movement

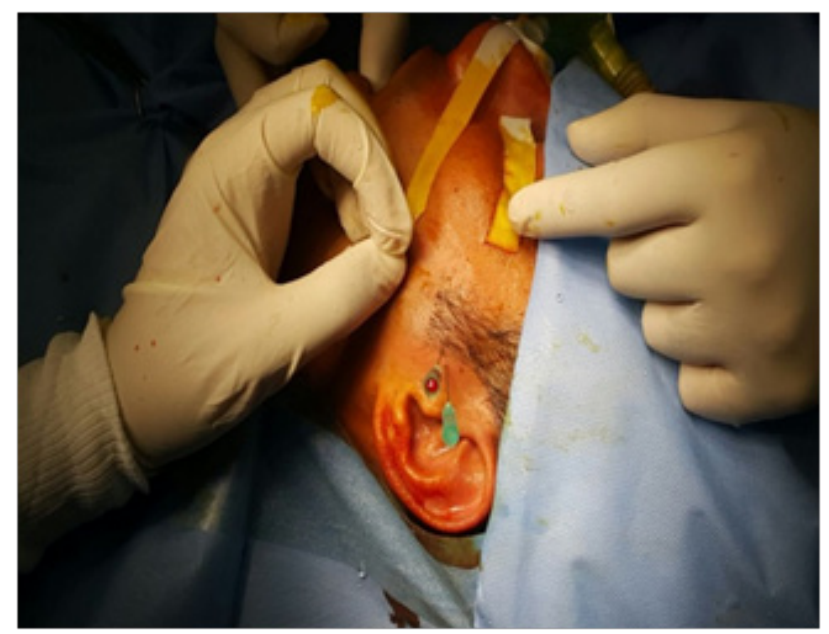

Fig. 1: inflammatory exudates outflow

\section{PRP preparation}

PRP was prepared by double centrifugation process. A small amount of blood $(10 \mathrm{cc})$ was obtained from the patient by venipuncture in acid citrate dextrose tubes (ACD) .

The blood was then centrifuged using a 'soft' spin (1500 rpm) for 10 minutes to separate RBCs from the remaining whole blood volume. After the first spin step, the blood was separated into three layers: an upper layer that contains mostly platelets and WBC, an intermediate thin layer that was known as the buffy coat and a bottom layer that consists mostly of RBCs.

For the production of pure PRP (P-PRP), upper layer (supernatant plasma) and superficial buffy coat were transferred to an empty sterile tube (without anticoagulant). Second spin step was then performed, it was centrifuged at a higher speed i.e a hard spin (3000rpm) for 10 minutes to obtain a platelet concentrate. PPP at the upper $2 / 3$ rd was then removed while PRP was obtained at lower $1 / 3 \mathrm{rd}$ of the tube by gently shaking the platelet pellets suspended in a minimum quantity of plasma .

At the end of the lavage procedure, the outflow needle was removed. Prefilled syringe with $2 \mathrm{ml}$ prp was mounted on the inflow needle and the solution was injected in the joint. All patients were then recalled at 1 and 6 months postoperatively.
A

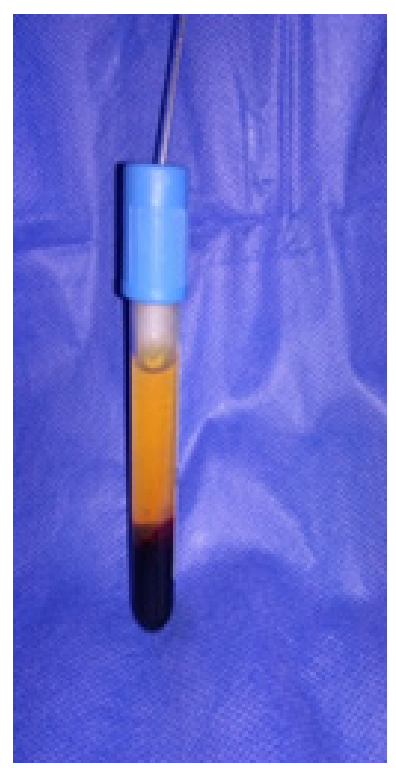

B

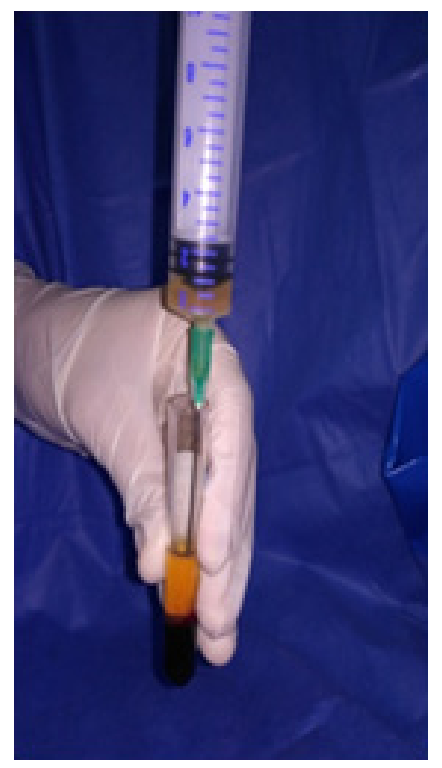

Fig. 2: A: centrifuged blood. B: plasma separation.

\section{Data management and analysis}

The collected data was revised,coded, tabulated and introduced to a pc using statistical package for social science. Analysis was done according to the type of data obtained for each parameter.

\section{RESULTS}

The study was conducted on 14 patients with a total of 20 joints ( 8 unilateral and 6 bilateral). They were randomly assigned into two groups using a simple unconstrained randomization method where each group contained seven patients. The 1st group which is the control group 
received arthrocentesis only, while the 2 nd group received arthrocentesis followed by PRP injection. Group (I) included seven patients; 3 females and 4 males, their age ranged from 19 to 30 years (with a mean of $23.143 \pm 4.180$ ). Group (II) included seven patients; 2 females and five males, their age ranged from 18 to 33 years (with a mean of $23.000 \pm 5.099$ ).

Regarding VAS for the included joints in both groups showed gradual decrease of pain by time. The lowest mean value was at 6 months postoperatively, while the greatest value was recorded pre-operatively. The statistical analysis of pain intensity (Fig. 3, Table 1) Paired T test revealed a significant difference between each two subsequent intervals. The difference between pre-operative mean value and the last post interval was statistically significant $(p<0.001)$ while comparing the two groups there was no statistically significant difference in the pain intensity between both groups during intervals period $(p>0.05)$.

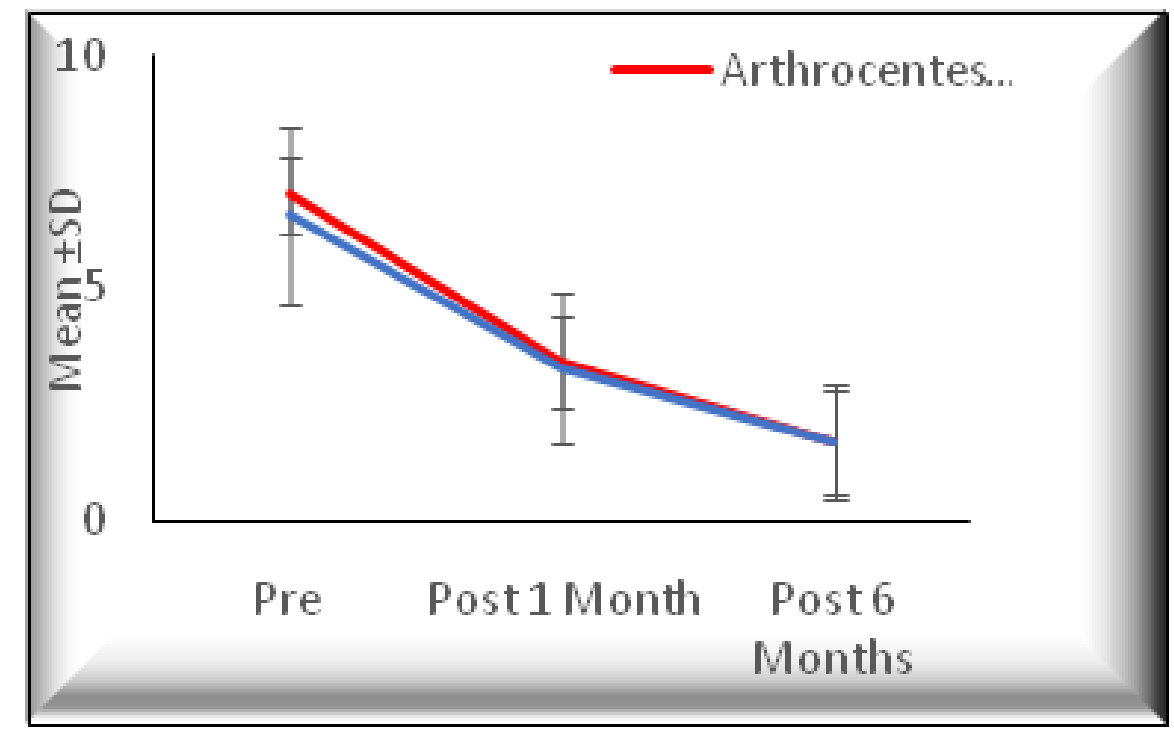

Fig. 3: Mean values of VAS pain in different groups

Table 1: Mean \pm SD of VAS pain values in both group and significance of the difference between each two intervals and between both groups using Paired t test

\begin{tabular}{|c|c|c|c|c|c|}
\hline \multicolumn{2}{|c|}{ VAS } & \multicolumn{2}{|c|}{ Groups } & \multicolumn{2}{|c|}{ T-Test } \\
\hline & & Arthrocentesis only & Arthrocentesis + PRP & $\mathrm{T}$ & $P$-value \\
\hline \multirow{2}{*}{ Pre } & Range & $6-8$ & $3-9$ & \multirow{2}{*}{0.548} & \multirow{2}{*}{0.594} \\
\hline & Mean \pm SD & $7.000 \pm 0.816$ & $6.571 \pm 1.902$ & & \\
\hline \multirow{2}{*}{ Post 1 month } & Range & $2-5$ & $1-6$ & \multirow{2}{*}{0.201} & \multirow{2}{*}{0.844} \\
\hline & Mean \pm SD & $3.429 \pm 0.976$ & $3.286 \pm 1.604$ & & \\
\hline \multirow{2}{*}{ Post 6 months } & Range & $0-3$ & $0-3$ & \multirow{2}{*}{0.000} & \multirow{2}{*}{1.000} \\
\hline & Mean \pm SD & $1.714 \pm 1.254$ & $1.714 \pm 1.113$ & & \\
\hline \multirow{2}{*}{$\mathrm{P}-1 \mathrm{M}$} & Differences & $3.571 \pm 0.535$ & $3.286 \pm 0.951$ & & \\
\hline & Paired Test & $<0.001^{*}$ & $<0.001 *$ & & \\
\hline \multirow{2}{*}{ P-6M } & Differences & $5.286 \pm 1.113$ & $4.857 \pm 1.069$ & & \\
\hline & Paired Test & $<0.001 *$ & $<0.001 *$ & & \\
\hline \multirow{2}{*}{$1 \mathrm{M}-6 \mathrm{M}$} & Differences & $1.714 \pm 0.951$ & $1.571 \pm 0.787$ & & \\
\hline & Paired Test & $0.003 *$ & $0.002 *$ & & \\
\hline
\end{tabular}

ns $=$ non-significant, $*$ statistically significant 
Regarding the maximum mouth opening, All patients showed gradual increase in maximum painless opening reaching their peak at 6 month. The mouth opening increased in both groups throughout the follow-up periods with no statistical significant difference $(p>0.05)$ between both groups (Fig. 4, Table 2).
The lateral movement of the affected side was measured in groups I and II. They were increased in group I and group II throughout the follow up periods, but the difference between the two groups throughout the whole follow-up period was found to be statistically insignificant $(p>0.05)$ (Table 3, Fig. 5).

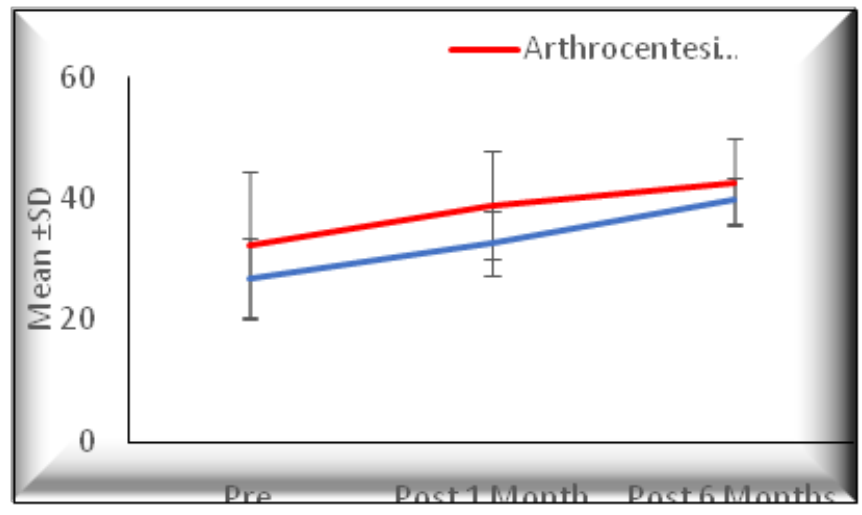

Fig. 4: Mean values of maximum painless opening in both groups

Table 2: Mean \pm SD of maximum mouth opening values in both group and significance of the difference between each two intervals and between both groups using Paired $t$ test

\begin{tabular}{|c|c|c|c|c|c|}
\hline \multicolumn{2}{|c|}{ MMO } & \multicolumn{2}{|c|}{ Groups } & \multicolumn{2}{|c|}{ T-Test } \\
\hline & & Arthrocentesis only & Arthrocentesis + PRP & $\mathrm{T}$ & $P$-value \\
\hline \multirow{2}{*}{ Pre } & Range & $20-50$ & $20-40$ & \multirow{2}{*}{1.073} & \multirow{2}{*}{0.304} \\
\hline & Mean \pm SD & $32.429 \pm 12.026$ & $26.857 \pm 6.644$ & & \\
\hline \multirow{2}{*}{ Post 1 month } & Range & $29-51$ & $26-41$ & \multirow{2}{*}{1.613} & \multirow{2}{*}{0.133} \\
\hline & Mean \pm SD & $39.000 \pm 8.794$ & $32.714 \pm 5.376$ & & \\
\hline \multirow{2}{*}{ Post 6 months } & Range & $32-53$ & $34-45$ & \multirow{2}{*}{0.996} & \multirow{2}{*}{0.339} \\
\hline & Mean \pm SD & $42.714 \pm$ & $39.714 \pm 3.684$ & & \\
\hline \multirow{2}{*}{$\mathrm{P}-1 \mathrm{M}$} & Differences & $-6.571 \pm 4.036$ & $-5.857 \pm 3.024$ & & \\
\hline & Paired Test & $0.005^{*}$ & $0.002 *$ & & \\
\hline \multirow{2}{*}{ P-6M } & Differences & $-10.286 \pm 6.499$ & $-12.857 \pm 5.305$ & & \\
\hline & Paired Test & $0.006^{*}$ & $<0.001 *$ & & \\
\hline \multirow{2}{*}{$1 \mathrm{M}-6 \mathrm{M}$} & Differences & $-3.714 \pm 3.498$ & $-7.000 \pm 3.464$ & & \\
\hline & Paired Test & $0.031^{*}$ & $0.002 *$ & & \\
\hline
\end{tabular}

$\mathrm{ns}=$ non-significant, $*$ statistically significant

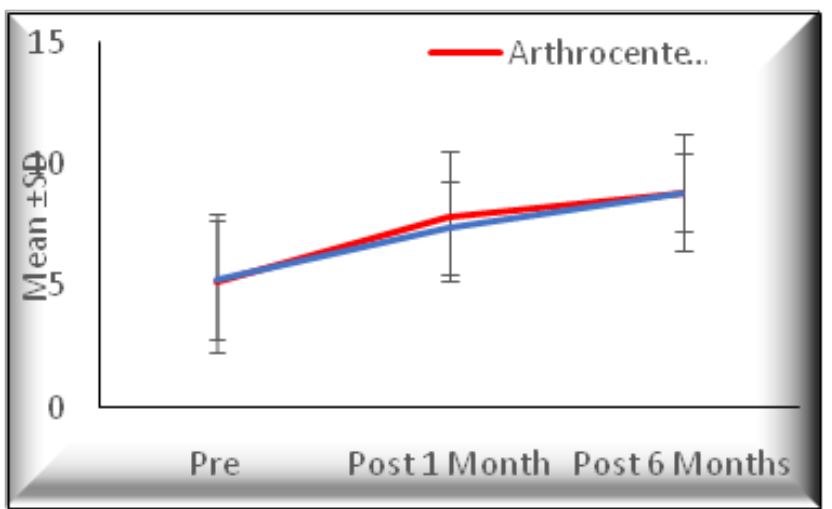

Fig. 5: Mean values of lateral excrusion movement of the affected joints in different groups 
Table 3: Mean \pm SD of lateral excrusion of the affected joint values in both group and significance of the difference between each two intervals and between both groups using Paired t test

\begin{tabular}{|c|c|c|c|c|c|}
\hline \multicolumn{2}{|c|}{ Lateral movement } & \multicolumn{2}{|c|}{ Groups } & \multicolumn{2}{|c|}{ T-Test } \\
\hline & & Arthrocentesis only & Arthrocentesis + PRP & $\mathrm{T}$ & $P$-value \\
\hline \multirow[b]{2}{*}{ Pre } & Range & $2-11$ & $2-10$ & \multirow[b]{2}{*}{-0.101} & \multirow[b]{2}{*}{0.921} \\
\hline & Mean \pm SD & $5.143 \pm 2.854$ & $5.286 \pm 2.430$ & & \\
\hline \multirow[b]{2}{*}{ Post 1 month } & Range & $-\quad 13$ & $5-11$ & \multirow[b]{2}{*}{0.346} & \multirow[b]{2}{*}{0.736} \\
\hline & Mean \pm SD & $7.857 \pm 2.673$ & $7.429 \pm 1.902$ & & \\
\hline \multirow{2}{*}{ Post 6 months } & Range & $6-13$ & $7-12$ & \multirow[b]{2}{*}{0.000} & \multirow[b]{2}{*}{1.000} \\
\hline & Mean \pm SD & $8.857 \pm$ & $8.857 \pm 1.574$ & & \\
\hline \multirow{2}{*}{$\mathrm{P}-1 \mathrm{M}$} & Differences & $-2.714 \pm 1.113$ & $-2.143 \pm 1.345$ & & \\
\hline & Paired Test & $0.001 *$ & $0.006^{*}$ & & \\
\hline \multirow{2}{*}{ P-6M } & Differences & $-3.714 \pm 1.254$ & $-3.571 \pm 1.272$ & & \\
\hline & Paired Test & $<0.001^{*}$ & $<0.001 *$ & & \\
\hline \multirow[b]{2}{*}{$1 \mathrm{M}-6 \mathrm{M}$} & Differences & $-1.000 \pm 0.577$ & $-1.429 \pm 0.787$ & & \\
\hline & Paired Test & $0.004^{*}$ & $0.003^{*}$ & & \\
\hline
\end{tabular}

ns= non-significant, *statistically significant

The lateral movement of the non affected side was measured in both groups. There were slight increase throughout the follow up periods. In the control group, paired $\mathrm{T}$ test revealed a significant difference between preoperative and 1 month postoperative mean value, while there was no significant difference between
1 month and 6 months postoperative subsequent intervals. In the study group, paired $\mathrm{T}$ test revealed no significant difference between each two subsequent intervals. There was no significance difference between the two groups at different observation times (Fig. 6, Table 4).

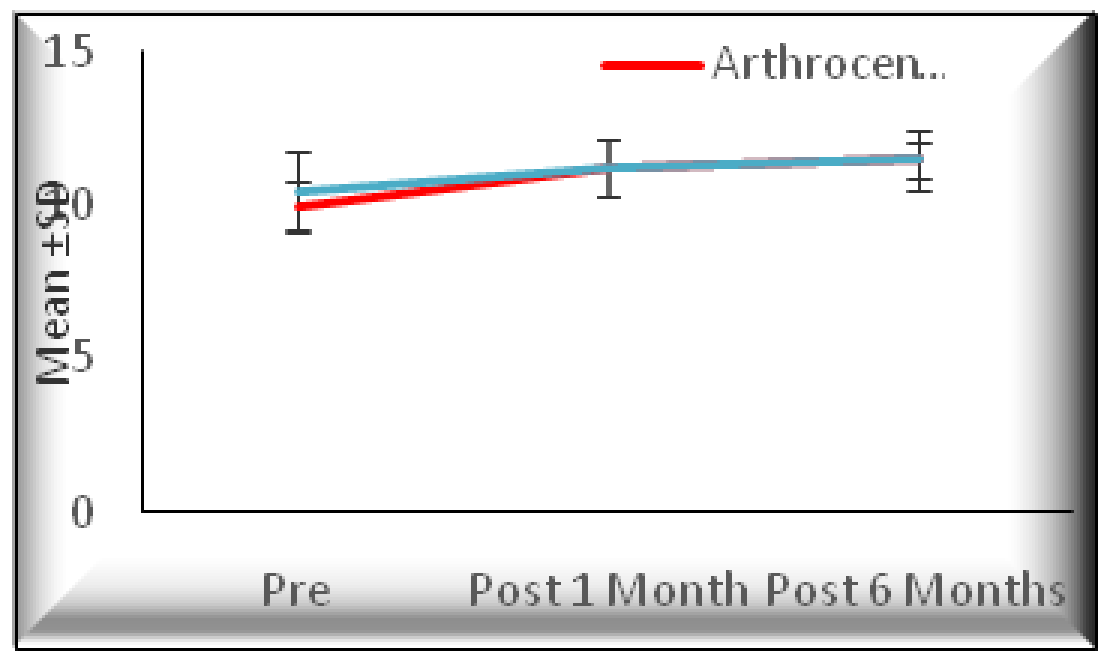

Fig. 6: Mean values of lateral excrusion movement of the non affected joints in different groups 
Table 4: Mean \pm SD of lateral excrusion of the non affected joints values in both group and significance of the difference between each two intervals and between both groups using Paired t test

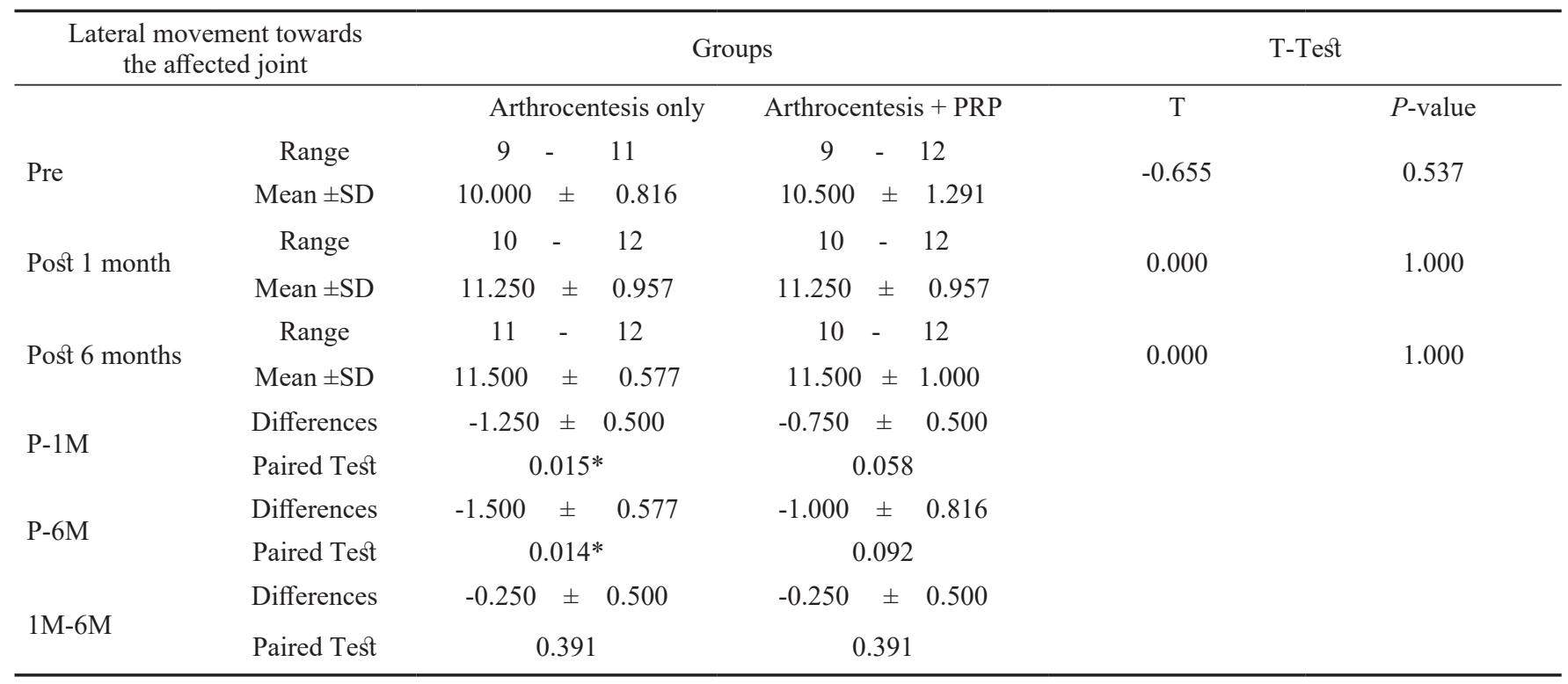

ns= non-significant, *statistically significant

Regarding protrusive movement in both groups, The lowest mean value was preoperatively recorded, while the greatest value was in PO-6 months. Paired T test revealed no significant difference between each two subsequent intervals, except for a significant difference in the interval between preoperative and PO-1 month $(p<0.001)$. Moreover, the difference between 1 and
6 month post operative interval was not statistically significant $(P=0.172)$, Table 5 , Fig 7). All patients showed slight improvement in protrusion after the 6 months follow up visit, while there was no significance difference between both groups throughout the whole follow-up period $(p>0.05)$.

Table 5: Mean \pm SD of protrusion values in both group and significance of the difference between each two intervals and between both groups using Paired $t$ test

\begin{tabular}{|c|c|c|c|c|c|}
\hline \multicolumn{2}{|c|}{ Protrusive movement } & \multicolumn{2}{|c|}{ Groups } & \multicolumn{2}{|c|}{ T-Test } \\
\hline & & Arthrocentesis only & Arthrocentesis + PRP & $\mathrm{T}$ & $P$-value \\
\hline \multirow{2}{*}{ Pre } & Range & $2.5-4$ & $2-4$ & \multirow{2}{*}{0.403} & \multirow{2}{*}{0.694} \\
\hline & Mean \pm SD & $3.286 \pm 0.567$ & $3.143 \pm 0.748$ & & \\
\hline \multirow{2}{*}{ Post 1 month } & Range & $3-4.5$ & $3-5$ & \multirow{2}{*}{0.206} & \multirow{2}{*}{0.841} \\
\hline & Mean \pm SD & $3.929 \pm 0.535$ & $3.857 \pm 0.748$ & & \\
\hline \multirow{2}{*}{ Post 6 months } & Range & $3.5-4.5$ & $3.5-5$ & \multirow{2}{*}{0.258} & \multirow{2}{*}{0.801} \\
\hline & Mean \pm SD & $4.071 \pm 0.450$ & & & \\
\hline \multirow{2}{*}{$\mathrm{P}-1 \mathrm{M}$} & Differences & $-0.643 \pm 0.244$ & $-0.714 \pm 0.267$ & & \\
\hline & Paired Test & $<0.001^{*}$ & $<0.001 *$ & & \\
\hline \multirow{2}{*}{ P-6M } & Differences & $-0.786 \pm 0.393$ & $-0.857 \pm 0.378$ & & \\
\hline & Paired Test & $0.002 *$ & $0.001^{*}$ & & \\
\hline \multirow{2}{*}{$1 \mathrm{M}-6 \mathrm{M}$} & Differences & $-0.143 \pm 0.244$ & $-0.143 \pm 0.244$ & & \\
\hline & Paired Test & 0.172 & 0.172 & & \\
\hline
\end{tabular}

ns $=$ non-significant, $*$ statistically significant 


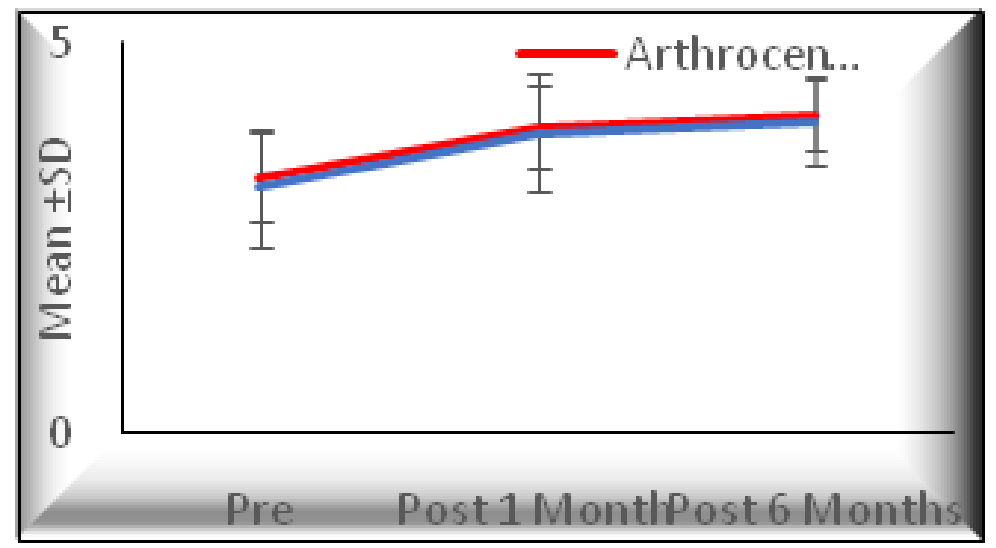

Fig. 7: Mean value of protrusion in both groups

\section{DISCUSSION}

The validity of arthrocentesis in the management of ID is a point of debate either alone or in conjunction with intra joint medication as PRP.

In our study, we assumed that arthrocentesis procedure followed by PRP injection will decrease pain and improve function by removing catabolites of inflammation by the action of lavage and PRP injection will remove pain inducers and provide a microenvironmental repair of disc, capsule, and retrodiscal pad. Consequently, our hypothesis was that arthrocentesis associated with PRP injection will be more effective in decreasing pain and improving function when compared to arthrocentesis done alone in patients with internal derangement. However, the results of this single blind randomized controlled trial found no evidence for a superior treatment effect on painful disc displacement without reduction (DDwoR) by using arthrocentesis and PRP injection compared to using arthrocentesis alone regarding pain intensity and physical functioning 6 months after treatment.

The landmarks we used for joint access during arthrocentesis were those adopted by Alkan et al.${ }^{[24]}$ which provided for easier access into the posterior recess of the joint, in contrary to those from the standard technique adopted by Nitzan et al. which was more difficult and results in more trauma to the joint due to repeated needle insertion to successfully engage the joint space. The superior joint space is used for arthrocentesis hence, it's larger and provides a better access for the joint as well as it the site in which the translatory movement occurs and also most adhesions are formed in the superior joint compartment.

TMJ lavage was performed by at least $150 \mathrm{ml}$ of lactated Ringer's solution to achieve the hydraulic effect necessary to release disc adhesions and at the same time efficiently remove the inflammatory and pain mediators ${ }^{[25]}$.
The results of our study revealed that all groups showed significant improvement in all the parameters under investigation including the pain, maximal painless opening, lateral excursions and protrusion, but without significant difference between the two groups. The improvement gradually increased starting from 1 month and up to 6 month.

This success is contributed to that the simple and minimally invasive lysis and lavage of the TMJ under hydraulic pressure tend to eliminate the vacuum effect and at the same time release the adhesions permitting a free movement of the articular disc during function and restoring the normal range of motion. It also helps in reducing the pain dramatically by washing out the inflammatory and pain mediators.

The use of additional intra joint medication PRP after arthrocentesis carry an extra value to the whole procedure outcome.

It has a potent anti-inflammatory effect, it focuses on the induction of functional recovery by means of regenerating weakened tissues, and its anabolic effect on synoviocytes lead to restore HA levels there by enhancing cartilage protection and joint lubrication ${ }^{[17,26,27,28,29]}$.

Although the reduction in VAS of pain was insignificant between the two groups. The reduction in pain in our study is in agreement with the results obtained by several authors $^{[17,29,30,31,32,33,34,35,36]}$ who reported an improvement in the pain levels in their studies after injection of PRP.

Pain decrease after injection of PRP was shown to be related to: early release of protease activated receptor 4 peptides from alpha granule in the platelets which has analgesic and anti-inflammatory effect lead to early relief of pain ${ }^{[17,29]}$.

The reduction in pain in the control group is 
in agreement with the results obtained by several authors ${ }^{[37,38]}$ who reported an improvement in the pain level post arthrocentesis in their studies by washing out of inflammatory mediators by arthrocentesis which had its effect in pain reduction and increasing range of movement.

The improvement in mandibular movement in the study group is in agreement with the results obtained by several authors ${ }^{[29,30,35,36,39,40,41]}$ who reported an increase in the measurements of mandibular movements including maximal interincisal opening, lateral and protrusive movements in their studies after injection of PRP.

Nitzan et al. $1991^{[37]}$ reported that arthrocentesis proved to be highly effective in providing significant improvement in pain reduction, maximal mouth opening and lateral movement toward the unaffected side in all patients. They claimed that physiotherapy following such treatment produced further improvement which comes in accordance with the results of the control group. In addition, many authors reported the improvement of mandibular functions following arthrocentesis ${ }^{[37,38]}$.

All patients in both groups had regional (joint and muscle) tenderness preoperatively which decreased gradually along the study period. The results were obtained in the study group as the same results were obtained in the other studies ${ }^{[29,30,34]}$. The results in the control group are in agreement with the results obtained by several authors ${ }^{[37,38,42]}$.

In the present study, when comparing the clinical outcomes to the preoperative values, both groups showed improvement in all the measured parameters, it was found to be insignificant thus, demonstrating the effectiveness of both methods in treatment of TMD patients.

\section{CONCLUSION}

The use of arthrocentesis alone or in conjunction with single intra articular injection of PRP were effective in reducing pain in cases of ID while there was no added therapeutic effect of single intra articular injection of PRP when used after arthrocentesis in cases of ID.

\section{CONFLICT OF INTEREST}

There are no conflicts of interest.

\section{REFERENCES}

1. DuBrul, EL. Sicher's oral anatomy. 7th ed. St. Louis (MO): C.V. Mosby; 1980. p. 146-61, 174-209.

2. Alomar X, Medrano J, Cabratosa J, Clavero JA, Lorente M, Serra I, et al. Anatomy of the temporomandibular joint. Semin Ultrasound CT MR 2007;28:170-183.

3. Nitzan DW, Marmary Y. The "anchored disc phenomenon": a proposed etiology for suddenonset, severe, and persistent closed lock of the temporomandibular joint. J Oral Maxillofac Surg 1997; 55:797-802; discussion 802-793.

4. Hoffmann RG, Kotchen JM, Kotchen TA, Cowley $\mathrm{T}$, Dasgupta $\mathrm{M}$, et al. Temporomandibular disorders and associated clinical comorbidities. Clin J Pain 2011;27: 268-274.

5. Dworkin SF, LeResche L. Research diagnostic criteria for temporomandibular disorders: review, criteria, examinations and specifications, critique. J Craniomandib Disord 1992;6:301-355.

6. Zakrzewska JM. Classification issues related to neuropathic trigeminal pain. J Orofac Pain 2004;18:325-331.

7. Wassell RW, Adams N, Kelly PJ. Treatment of temporomandibular disorders by stabilising splints in general dental practice: results after initial treatment. $\mathrm{Br}$ Dent J 2004;197:35-41; discussion 31

8. Sidebottom AJ. Current thinking in temporomandibular joint management. Br J Oral Maxillofac Surg 2009; 47: 91-4.

9. Emes Y, Arpinar IS, Oncu B, Aybar B, Aktas I, Al Badri $\mathrm{N}$, et al. The next step in the treatment of persistent temporomandibular joint pain following arthrocentesis: A retrospective study of 18 cases. J Craniomaxillofac Surg 2013;42:e65-e69.

10. Murakami K. Rationale of arthroscopic surgery of the temporomandibular joint. Journal of oral biology and craniofacial research2013; 3:126-134.

11. Grossmann E .Arthrocentesis techniques applied to arthrogenic temporomandibular joint disorders Rev Dor. São Paulo2012; 13:374-381.

12. Tvrdy P, Heinz P, Pink R .Arthrocentesis of the temporomandibular joint: A review. Biomed Pap Med Fac Univ Palacky Olomouc Czech Repub2013; 159:31-34

13. Bielecki TM, Gazdzik TS, Arendt J, Szczepanski T, Krol W, Wielkoszynski T. Antibacterial effect of autologous platelet gel enriched with growth factors and other active substance: An in vitro study. J Bone Joint Surg Br 2007;89:417-420. 
14. Napolitano M, Matera S, Bossio M, Crescibene A, Costabile E, et al. Autologous platelet gel for tissue regeneration in degenerative disorders of the knee. Blood Transfus2012; 10: 72-77.

15. Prakash S, Thakur A .Platelet concentrates: past, present and future. J Maxillofac Oral Surg 2011;10: 45-49.

16. Carlson NE, Roach RB Jr .Platelet-rich plasma: clinical applications in dentistry. J Am Dent Assoc 2002;133: 1383-1386.

17. van Buul GM, Koevoet WL, Kops N, Bos PK, Verhaar JA, et al. Platelet-rich plasma releasate inhibits inflammatory processes in osteoarthritic chondrocytes. Am J Sports Med 2011; 39: 2362-2370.

18. Lippross S, Moeller B, Haas H, Tohidnezhad M, Steubesand $\mathrm{N}$, et al. Intraarticular injection of platelet-rich plasma reduces inflammation in a pig model of rheumatoid arthritis of the knee joint. Arthritis Rheum2011; 63: 3344-3353.

19. Song SU, Cha YD, Han JU, Oh IS, Choi KB, Lee JW et al. Hyaline cartilage regeneration using mixed human chondrocytes and transforming growth factor beta-1 producing chondrocytes. Tissue Eng 2005; 11:1516-1526.

20. Noth U, Rackwitz L, Heymer A, Weber M, Baumann B, Steinert A et al .Chondrogenic differentiation of human mesenchymal stem cells in collagen type I hydrogel. J Biomed Mater Res A2007; 83:626-635.

21. Schmidt MB, Chen EH, Lynch SE .A review of effects of insulin like growth factor and platelet derived growth factor on in vivo cartilage healing and repair. Ostoarthr Cartil2006; 14:403-412.

22. Martin JA, Buckwalter JA.The role of chondrocytematrix interaction in maintaining and repairing articular cartilage. Biorheology2000; 37:129-140.

23. O' Keefe RJ, Crabb ID, Puzas JE, Rosier RN. Effects of transforming growth factor-beta 1 and fibroblast growth factor on DNA synthesis in growth plate chondrocytes are enhanced by insulin like growth factor-1. J Orthop Res 1994;12;299. 310.

24. Alkan A, Etöz OA: A new anatomical landmark to simplify temporomandibular joint arthrocentesis. Br J Oral Maxillofac Surg.July 2009.

25. Kaneyama K, Segami N and Nishmura
M: The ideal lavage volume for removing bradykinin, interleukin-6, and protein from the temporomandibular joint by arthrocentesis. J Oral Maxillofacial Surg,2004; 62:657-661.

26. Akeda K, An H, Okuma M, Attawia M, Miyamoto K, Thonar E, et al. Platelet-rich plasma stimulates porcine articular chondrocyte proliferation. Osteoarthritis and Cartilage Journal 2006; 14:1272-80.

27. 27. Xie X, Zhang C, Tuan RS. Biology of plateletrich plasma and its clinical application in cartilage repair and matrix biosynthesis. Arthritis Research and Therapy 2014; 16:204-19.

28. Mazzocca AD, Mc Carthy $\mathrm{BR}$, Intravia J, Beitzel K, Apostolakos J, Cote MP, et al. An in vitro evaluation of the anti-inflammatory effects of platelet-rich plasma, ketorolac, and methyl prednisolone. J Arthroscopy 2013; 29:675-83.

29. Machoň V, Řehořová M, Šedý J, Foltán R. Platelet-Rich Plasma in Temporomandibular Joint Osteoarthritis Therapy: A 3-Month Follow-Up Pilot Study. J Arthritis 2013; 2: 112.

30. Pihut M, Szuta M, Ferendiuk E, Zeńczak, Więckiewicz D. Evaluation of Pain Regression in Patients with Temporomandibular Dysfunction Treated by Intra-Articular Platelet-Rich Plasma Injections: A Preliminary Report. Biomed Res Int 2014; 2014:132369.

31. Chomicki P, Zakrzewski P, Pomianowski S, Bindas P. Platelet concentrates, as new and promising agent in the orthopedic surgery- an introduction. Progres of Medical Science 2010; 23: 153-7.

32. Ficek K, Ficek A. Platelet rich plasma application in a bone loss case. Medical Cases 2013; 4: 175-9.

33. Iwanaga $T$, Shikichi $M$, Kitamura $H$, Yanase $H$, Nozawa-Inoue K. Morphology and functional roles of synoviocytes in the joint. Arch Histol Cytol. 2000; 63:17-31.

34. Hegab AF, Ali HE, Elmasry M, Khallaf MG. Platelet-Rich Plasma Injection as an Effective Treatment for Temporomandibular Joint Osteoarthritis. J Oral Maxillofac Surg 2015; 73:1706-13

35. Hanci M, Karamese M, Tosun Z, Aktan T, Duman $\mathrm{S}$, Savaci N. Intra-articular platelet-rich plasma injection for the treatment of temporomandibular disorders and a comparison with arthrocentesis. J Craniomaxillofac Surg 2015; 43:162-6. 
36. Moon S, Lee S, Ryu J. Ultrasound-guided Plateletrich Plasma Prolotherapy for Temporomandibular Disorders. J Oral Med Pain 2014; 39: 140-5.

37. Nitzan DW, Dolwick MF, Martinez GA. Temporomandibular joint arthrocentesis: a simplified treatment for severe, limited mouth opening. J Oral Maxillofac Surg 1991; 49:1163-1167; discussion 1168-1170.

38. EL-said S, Shawky N, RagaeyH.Comparative study of arthrocentesis with or without using piroxicam in the management of temporomandibular joint disorders. Theses for master degree in Oral and Maxillofacial Surgery. Alexandria University, 2015.

39. Simsek M. Bilateral platelet rich plasma injections with assisted techniques for temporomandibular joint disorders. Eur Res J 2016; 2: 42-5.

40. Cerza F, Carnì S, Carcangiu A, Di Vavo I, Schiavilla V, Pecora, A, et al. Comparison between hyaluronic acid and platelet-rich plasma, intra-articular infiltration in the treatment of gonarthrosis. Am. J. Sports Med 2012; 40: 2822-7.

41. Sharma S.M, Dhruvit T. clinical efficacy of autologous concentrate platelets in treatment of TMJ disorders- apilot study. Nitte University J of Health Science 2014; 4:70-4.

42. ELNager Y, Shawky N, Ragaey H, Abd Ellatief $G$. the study of the effect of arthocentesis on muscle activiy using electromyogram. Theses for master degree in Oral and Maxillofacial Surgery. Alexandria University, 2010. 